\title{
Aproximación bibliométrica a la Revista de Comunicación (Perú), 2002-2019
}

\section{A bibliometric approach to Revista de Comunicación (Peru) since 2002 to 2019}

\author{
Estrada-Cuzcano, A., Alhuay-Quispe, J. ${ }^{1}$ \\ Recibido: 28-04-2020 - Aceptado: 20-08-2020 \\ https://doi.org/10.26441/RC19.2-2020-A6
}

RESUMEN: El estudio presenta un análisis de visibilidad e impacto científico de los artículos aplicados por la Revista de Comunicación entre 2002 a 2019. Los métodos y técnicas empleados son de enfoque bibliométrico a nivel de autor (Lotka, índice de colaboración), artículo (co-ocurrencia) y revista (h-index, citas). Se emplean las fuentes de datos de citas Scopus, Web of Science y Google Scholar, y dos herramientas de análisis: Publish or Perish y VOSviewer. Los resultados encontrados muestran un desempeño óptimo de la Revista de Comunicación que evidencia su posicionamiento como una de las mejores publicaciones seriadas indizadas de América Latina y España que integra un selecto segmento de revistas de comunicación.

Palabras clave: revistas científicas; revistas peruanas; indicadores de evaluación; bibliometría.

ABSTRACT: This article presents a visibility and scientific impact analysis based on papers published by Revista de Comunicación since 2002 to 2019. Bibliometric methods used included approach at authorlevel (Lotka, collaboration index), article-level (co-occurrence) and journal-level (h-index, citations). Also, Scopus, Web of Science and Google Scholar were used as citation data sources, Publish or Perish and VOSviewer as analysis tools. Results shows an optimal performance from Revista de Comunicación that evidences its best positioning between indexed serial publications from Latin America and Spain wich integrate a select group of scholarly journals in communication.

Keywords: scholarly journals; peruvian scientific journals; research evaluation indicators; bibliometrics.

\footnotetext{
${ }^{1}$ Alonso Estrada-Cuzcano es Doctor en Documentación: archivos y bibliotecas en el entorno digital por la Universidad Carlos III de Madrid, miembro del Grupo de Investigación Tecnologías, derecho y libertades informativas. Investigador RENACYT-Concytec (P0024402). Docente principal en la Escuela Profesional de Bibliotecología y Ciencias de la Información de la Universidad Nacional Mayor de San Marcos. mestradac@unmsm.edu.pe, https:// orcid.org/0000-0001-5039-1108

Joel Alhuay-Quispe es Licenciado en Bibliotecología y Ciencias de la Información (UNMSM), con maestría en Gestión de la Información y del Conocimiento. Investigador RENACYT-Concytec (P0023249). Universidad Nacional Mayor de San Marcos, Vicerrectorado de Investigación y Postgrado, bibliómetra y consultor en evaluación de revistas científicas. joel.alhuay@unmsm.edu.pe, https://orcid.org/0000-0002-1903-4687
} 


\section{Introducción}

Las revistas son el principal medio para la difusión de la comunicación científica, en la actualidad tienen un crecimiento sostenido impulsado por la aparición de tecnologías emergentes que posibilitaron la creación de herramientas de gestión editorial (Yance-Yupari, 2018) aunada a la aparición del movimiento de acceso abierto (open access). De acuerdo con Alperin y Rozemblum (2017), la evolución de las revistas latinoamericanas se categorizan en tres etapas: 1) emergente (1985 - 1995), antes de esa fecha no existía un número consolidado de revistas, se hacen explícitas algunas problemáticas y se avizora el impacto de los soportes electrónicos; 2) consolidación de las revistas (1995 - 2015), se le denomina la "edad de oro" de las iniciativas regionales (Latindex, SciELO y Redalyc), y 3) "internacionalización" de las revistas (2015 - presente), después de avances en la "regionalización" de la ciencia se evidencia cambios en las políticas de los gobiernos en el fomento de las revistas que alcanzan un índice de citación en bases de datos internacionales como Scopus o WoS, entre otros indicadores.

En el caso peruano, las revistas académicas responden a características propias, según Santillán-Aldana et al. (2017) "las organizaciones editoras corresponden, en su mayoría (73.9\%), a universidades, dos tercios de las cuales son de carácter privado"; las revistas peruanas mantienen un formato hibrido, un $67 \%$ aproximadamente mantiene los formatos electrónicos e impresos, mientras un $20 \%$ publica en formato electrónico y un 13\% en impreso. Los formatos electrónico e impreso fueron utilizados por la Revista de Comunicación hasta el año 2019, ahora sólo se publica en digital.

Las revistas son una referencia en campo académico y "en este contexto el investigador se ha convertido en autor, lector, evaluador y editor de su propia disciplina, y también una influencia para la sociedad" (Alperin \& Rozemblum, 2017); así mismo, las citaciones se consideran indicadores que reflejan de algún modo la trascendencia del artículo en su entorno de publicación y proyecta el prestigio del autor. Las citaciones hechas por los autores denotan la influencia, experiencia y prioridades en la elección de las revistas más importantes donde publicar (Fernández-Quijada, 2011, p. 100). Las referencias tienen una multitud de funciones en el artículo científico, solo se cita una pequeña proporción de la literatura relevante y los autores tienen una multitud de motivos para incluir estudios particulares como referencias (Aksnes et al., 2019).

Las revistas científicas se requieren de análisis bibliométricos, porque la medición o cuantificación de los datos obtenidos de la propia comunicación académica se utilizan para explicar, entender o interpretar algunos procesos causales de la publicación (Borgman \& Furner, 2002). La evaluación de revistas, a partir de indicadores bibliométricos, permite la toma de decisiones basadas en criterios de valor necesarios en la gestión y posicionamiento de la revista. Existen trabajos de análisis bibliométricos realizados para una revista en particular o para revistas agrupadas por algún criterio temático, temporal o geográfico, entre otros.

Se revisan algunos ejemplos de artículos que reseñan análisis bibliométricos que aplican diversas metodologías; Shukla y Verma (2019) realizaron un análisis de la revista Journal of Knowledge and Communication Management por 7 años donde estudiaron patrones de autoría (múltiple y autoría única) y productividad de autores en el periodo 2011-2017; Lochan Jena et al. (2012), analizan al Journal of Financial Crime en un periodo de cinco años, entre los indicadores empleados están la cantidad de autores por documentos, evolución cronológica de la citas recibidas; Laengle et al. (2017), toman como referencia los cuarenta años de la revista European Journal of Operational Research basados en datos de la Web of Science Core Collection, analizan términos de las palabras clave del autor para los análisis de co-ocurrencia y visualización usando VOSviewer. Los indicadores que usan son números de documentos, citaciones, citas por documento, $h$-index.

Reeves y Borgman (2006) estudiaron un grupo de nueve revistas en el campo de la comunicación para evaluar el tipo de material citado, frecuencia de las citas y las autocitas, y determinar su 
influencia entre las revistas dentro y fuera del campo, se toman los indicadores de Social Sciences Citation Index aparecidos en Journal Citation Reports, correspondientes a 1977-1979.

Es preciso examinar, a partir de una revisión panorámica, los criterios de evaluación y permanencia de revistas en los índices internacionales, los indicadores bibliométricos, a nivel de revista y por ende a nivel del autor y artículos; utilizadas como herramientas necesarias para mantener y mejorar la calidad editorial, impacto científico y visibilidad de una revista en la actualidad.

Sin embargo, aun cuando en años recientes hay un crecimiento en publicaciones inadecuadamente llamadas "estudios bibliométricos", estos no necesariamente emplean los enfoques y técnicas basados en estudios métricos de la información (i.e. bibliometría) de manera rigurosa y exhaustiva; una de las razones radica en que estos profesionales (autores) no siempre tienen la formación necesaria o las capacidades específicas para utilizar las herramientas bibliométricas adecuadamente (Vílchez-Román \& Alhuay-Quispe, 2016).

\section{Sobre la Revista de Comunicación, Piura}

Tabla1. Revistas de comunicación de América Latina y España indizadas en WoS y Scopus

\begin{tabular}{|c|c|c|c|c|c|c|c|}
\hline $\mathbf{N}^{\circ}$ & Título & 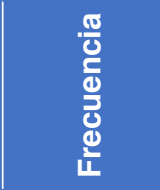 & $\frac{\varphi}{\bar{c}}$ & ต & $\begin{array}{l}\text { 号 } \\
\text { P } \\
\text { I }\end{array}$ & $\begin{array}{l}\infty \\
0 \\
\vdots \\
\vdots \\
1\end{array}$ & 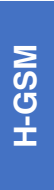 \\
\hline 1 & Anàlisi. Quaderns de Comunicació i Cultura & Bianual & España & $\begin{array}{l}\text { SCO, } \\
\text { ESCI }\end{array}$ & 3 & 2 & 8 \\
\hline 2 & Brazilian Journalism Research & Semestral & Brasil & $\begin{array}{l}\text { SCO, } \\
\text { ESCI }\end{array}$ & 1 & 2 & 8 \\
\hline 3 & Communication \& Society & Trimestral & España & $\begin{array}{l}\text { SCO, } \\
\text { ESCl }\end{array}$ & 13 & 6 & 19 \\
\hline 4 & Comunicar & Trimestral & España & $\begin{array}{l}\text { SCO, } \\
\text { SSCI }\end{array}$ & 32 & 18 & 39 \\
\hline 5 & Cuadernos.info & Semestral & Chile & $\begin{array}{l}\mathrm{SCl}, \\
\mathrm{SCO}, \\
\mathrm{ESCl}\end{array}$ & 8 & 6 & 13 \\
\hline 6 & El Profesional de la información & Bimestral & España & $\begin{array}{l}\text { SCO, } \\
\text { SSCI }\end{array}$ & 25 & 14 & 28 \\
\hline 7 & Historia y comunicación social & Semestral & España & $\begin{array}{l}\mathrm{SCO}, \\
\mathrm{A} \& \mathrm{H}- \\
\mathrm{Cl}\end{array}$ & 7 & 3 & 15 \\
\hline 8 & IC: Revista Científica de Información y Comunicación & Anual & España & $\begin{array}{l}\text { SCO, } \\
\text { ESCI }\end{array}$ & 1 & 1 & 4 \\
\hline 9 & Icono14 & Semestral & España & $\begin{array}{l}\text { SCO, } \\
\text { ESCI }\end{array}$ & 1 & 6 & 13 \\
\hline 10 & Palabra Clave & Semestral & Colombia & $\begin{array}{l}\mathrm{SCl}, \\
\mathrm{SCO}, \\
\mathrm{ESCl}\end{array}$ & 8 & 5 & 15 \\
\hline 11 & Revista de Comunicación & Semestral & Perú & $\begin{array}{l}\mathrm{SCl}, \\
\mathrm{SCO}, \\
\mathrm{ESCl}\end{array}$ & 1 & 2 & - \\
\hline 12 & Revista Latina de Comunicación Social & Anual & España & $\begin{array}{l}\text { SCO, } \\
\text { ESCI }\end{array}$ & 14 & 8 & 29 \\
\hline 13 & Trípodos & Semestral & España & $\begin{array}{l}\text { SCO, } \\
\text { ESCl }\end{array}$ & 1 & 3 & 11 \\
\hline
\end{tabular}

Nota: A\&HCI - Arts \& Humanities Citation Index; ESCI - Emerging Source Citation Index; SCI - SciELO; SCO: Scopus; $\mathbf{S S C I}$ - Social Sciences Citation Index. Fuente: elaboración propia. 
El primer número de la Revista de Comunicación aparece en el año 2002 y se presenta como el inicio de una "nueva etapa de trabajo académico, orientada a impulsar estudios y propuestas para la mejora del pensamiento y trabajo profesional en comunicación. Con ese ánimo, la revista crea un espacio para promover la reflexión y discusión" (Vargas Bianchi, 2002); mantiene una periodicidad anual hasta el año 2016, y a partir del año 2017 cambia su periodicidad a semestral. La Revista de Comunicación ha sido parte de algunos estudios sobre impacto, calidad o visibilidad de revistas a nivel internacional (Castillo-Esparcia, 2012; Castillo Esparcia et al., 2012; Chávez-Sánchez, 2020) o estudios de revistas a nivel nacional (Morales Morante, 2016).

La Revista de Comunicación se encuentra indizada en las tres plataformas multidisciplinarias de mayor prestigio internacional WoS-ESCI (2019), Scopus (2018) y SciELO (2018), esta última se considera la base de datos de mayor trascendencia en Iberoamérica. Sólo un número reducido y selecto de revistas de comunicación en América Latina y España (13 en total) están indizadas en Scopus y WoS (Tabla 1). Las revistas españolas tienen una presencia mayor que las revistas latinoamericanas que se han incorporado en las bases de datos antes mencionadas. Algunas de las revistas aún no tienen indicadores en Journal of Citation Report (JCR) y SCImago Journal \& Country Rank (SJR) o Google Scholar Metrics (GSM), ni sus respectivos cuartiles. La ausencia de indicadores se debe a la reciente incorporación de revistas a las bases de datos antes mencionadas, como es el caso de la Revista de Comunicación (Piura). Los cuartiles son indicadores que se utilizan para determinar el impacto o calidad de las revistas científicas (Sáez \& Barranquero Carretero, 2019; Vizoso et al., 2019).

La Revista de Comunicación posee Índice H en JCR, SJR y GSM en escalas que van desde el 1 a 5. El Índice $\mathrm{H}$ tiene algunas limitaciones pero es aplicado frecuentemente para determinar el impacto de un autor o de las publicaciones. La irrupción del GSM frente al duopolio de WoS y Scopus genera cambios importantes en los indicadores bibliométricos porque tiene una mayor cobertura e índices más elevados, tal como lo demuestra el estudio sobre revistas de comunicación de (Delgado \& Repiso, 2013).

\section{Métodos}

\section{Recuperación y fuentes de datos}

Los metadatos de los artículos extraídos del portal web de la revista (https://revistadecomunicacion.com) fueron los siguientes: autores, título, volumen, número, año. Los artículos identificados $(n=291)$ corresponden a todos los publicados por la revista desde sus inicios en el 2002 hasta el 2019, incluyendo todos los tipos de secciones de la revista: editorial, artículos, reseñas.

El motor de búsqueda Google Scholar (GS), a partir del programa informático Publish or Perish, fue utilizado como fuente para la recuperación de las citas recibidas por los artículos publicados por la revista en el periodo de estudio. Las bases de datos de literatura científica de acceso por suscripción Scopus y Web of Science fueron utilizadas como fuentes adicionales para la recuperación de citas a los artículos analizados. A partir de las tres fuentes, se consultan los datos de citaciones recibidas por artículos publicados por la revista en todo el periodo analizado. Se emplea una cadena de búsqueda para cada fuente de datos con la siguiente característica:

- GS: "source" + "issn" + "year"

- Scopus: REFSRCTITLE en búsqueda avanzada.

- WoS: "Cited work" en la interfazcited reference search.

- La recolección de los datos se realizó en noviembre del 2019. 
Procesamiento y análisis de los datos

Para la extracción de las co-ocurrencias de términos se usaron los títulos de los 291 artículos. El pre-procesamiento del corpus (titles) arrojó 528 palabras, a partir de los que se identificaron términos susceptibles de normalización usando un tesauro construido ad hoc de 141 palabras, que contenían términos topónimos y sinónimos.

Los indicadores bibliométricos empleados se basaron a nivel del artículo y del autor:

Estimación Lotka. - Lotka formula la ley inversa del cuadrado de la productividad científica basado en los datos analizados de la productividad de autores en Chemical Abstracts y Auerbachs. Para Lotka, la ecuación que permite estimar la mejor relación entre la productividad de los autores y el número de firmas sería: $x{ }^{n}{ }^{n} y=\mathrm{C}$, despejando la variable " $\mathrm{y}$ " se formula como:

$$
\mathrm{y}=\frac{\mathrm{c}}{\mathrm{x}^{\mathrm{n}}}
$$

donde:

y es el número de autores que producen "x" artículos.

C es el número de autores observados que realizan una sola aportación.

$\mathrm{X}$ es la producción científica o número de artículos producidos.

$\mathrm{n}=2$.

Índice de Colaboración. - La extensión de la colaboración en las ciencias sociales y humanidades, o índice de Colaboración (IC) se calcula mediante el número medio de autores por artículo (Urbizagastegui Alvarado, 2011) que se representa por la siguiente ecuación:

$$
I C=\frac{\sum_{j=1}^{n} j f_{j}}{N}
$$

Donde:

IC es el índice de colaboración

$j f_{j}$ es el número de publicaciones en colaboración durante un determinado periodo $\mathbf{j}$.

$\mathrm{N}$ es el número total de documentos publicados durante el mismo determinado periodo.

Citas por documento. - Relación entre la cantidad de citas recibidas en determinado año "a" por el total de documentos publicados en ese mismo año.

$$
\text { CitDoc }=\frac{\text { Cit }_{a}}{\operatorname{Doc}_{a}}
$$

Donde:

$\mathrm{Cit}_{\mathrm{a}}$ es el total de citas recibidas en el año "a".

$\operatorname{Doc}_{\mathrm{a}}$ es el número de artículos publicados en el año "a".

$a$ es el año o periodo temporal de corte. 
Citas recibidas por autor. - Cociente de la cantidad de citas recibidas en determinado año "b" por el total de firmas de autor de los artículos publicados en ese mismo año.

Donde:

$$
\text { CitDoc }=\frac{\text { Cit }_{b}}{\text { Fir }_{b}}
$$

$\mathrm{Cit}_{\mathrm{b}}$ es el total de citas recibidas en el año " $\mathrm{b}$ ",

Fir $_{b}$ es el total de firmas de autores en el año " $b$ ".

$b$ es el año o periodo temporal de corte.

Índice de Hirsch.- El índice $H$ fue definido por Jorge Hirsch como el número de trabajos con número de citación $\geq \mathrm{h}$, propuesto para caracterizar la producción científica de un investigador (Hirsch, 2005). Se calcula a partir del número de artículos publicados por un autor y las citas de esos trabajos, por ejemplo, si un autor tiene un índice h de 5, entonces publicó en promedio cinco trabajos, cada uno de los cuales ha sido citado en otros trabajos al menos 5 veces (Vílchez-Román, 2014). El modelo lineal que define este indicador corresponde a la siguiente distribución:

$$
N_{c}(y)=N_{0}-\left(\frac{N_{0}}{h}-1\right) y
$$

Donde:

$\mathrm{N}_{\mathrm{c}}(y)$ es el número de citaciones del $y$-enésimo artículo ordenado del más citado al menos citado.

$\mathrm{N}_{0}$ es el número de citas del documento más citado.

Co-ocurrencia de términos. - De acuerdo con (He, 1999), un análisis de frecuencia de términos (frecuencias $=k$ ) consiste en la extracción de términos o palabras clave a partir de un conjunto de registros documentales provenientes de bases de datos, pero también de un documento textual, a partir de los cuales se construye una matriz de palabras clave. El análisis de co-ocurrencia de términos se realizó a partir de los títulos de los artículos en inglés como idioma de entrada de datos dado que el programa empleado VOSviewer (www.vosviewer.com) analiza el corpus con mayor precisión en inglés por ser este el idioma del desarrollo del aplicativo (Van Eck \& Waltman, 2020). Los términos que conformaron el corpus fueron seleccionados por el método de conteo binario (binary counting) que mide la presencia o ausencia de un término en un registro. El umbral empleado como cantidad límite inferior de ocurrencias por término fue de dos palabras.

\section{Resultados}

\section{Productividad, firmas y citas de autores}

En la investigación se consideran autores más productivos a quienes publicaron tres o más artículos en la Revista de Comunicación, los cuales alcanzan un número de 19 (Tabla 2). Entre los autores más productivos se encuentran algunos procedentes de la propia Universidad de Piura (UDEP) o en todo caso tuvieron alguna vinculación con la universidad. En la Tabla 2 se muestra en las cuatro primeras ubicaciones a los autores más productivos con 9 a 7 artículos publicados, que representa el $10.65 \%$ del total de artículos ( $\mathrm{n}=291$ ), que tienen como característica su vinculación con la universidad por la procedencia de los estudios universitarios o por su función docente. 
Tabla 2. Autores con mayor productividad y citas

\begin{tabular}{|l|c|c|c|c|c|}
\multirow{2}{*}{\multicolumn{1}{|c|}{ Autor }} & \multicolumn{2}{c|}{ Docs. } & \multicolumn{2}{c|}{ Citas } \\
\cline { 2 - 6 } & $n$ & $\%$ & GS & Sco & WoS \\
\hline Requejo Alemán, J. & 9 & 3,09 & 14 & 1 & 1 \\
\hline Vargas Bianchi, L. & 8 & 2,75 & 1 & 1 & 1 \\
\hline Arbaiza Rodríguez, F. & 7 & 2,41 & 27 & 0 & 3 \\
\hline Atarama Rojas, T. & 7 & 2,41 & 20 & 4 & 4 \\
\hline Herrera Damas, S. & 5 & 1,72 & 33 & 4 & 5 \\
\hline Ramos Rugel, R. & 5 & 1,72 & 1 & 0 & 0 \\
\hline Salazar Velarde, M. & 5 & 1,72 & 5 & 1 & 0 \\
\hline Yezers'ka, L. & 5 & 1,72 & 7 & 1 & 1 \\
\hline Zeta De Pozo, R. & 5 & 1,72 & 10 & 2 & 0 \\
\hline Gutiérrez Rentería, M. & 4 & 1,37 & 11 & 4 & 1 \\
\hline Salas Andrade, N. & 4 & 1,37 & 2 & 0 & 0 \\
\hline Campos-Freire, F. & 3 & 1,03 & 14 & 2 & 2 \\
\hline Huamán Flores, F. & 3 & 1,03 & 2 & 0 & 0 \\
\hline Mauricio Trelles, I. & 3 & 1,03 & 0 & 0 & 0 \\
\hline Restrepo, J. & 3 & 1,03 & 15 & 0 & 0 \\
\hline Robledo Dioses, K. & 3 & 1,03 & 0 & 0 & 0 \\
\hline Rodríguez Rodríguez, J. & 3 & 1,03 & 0 & 0 & 0 \\
\hline Sánchez-Escalonilla, A. & 3 & 1,03 & 2 & 0 & 0 \\
\hline Velásquez, R. & 3 & 1,03 & 1 & 0 & 0 \\
\hline Total & $\mathbf{2 9 1}$ & $\mathbf{1 0 0}$ & $\mathbf{9 8 7}$ & $\mathbf{1 6 9}$ & $\mathbf{1 5 9}$ \\
\hline
\end{tabular}

Fuente: elaboración propia. Nota: GS: Google Scholar, Sco: Scopus, WoS: Web of Science.

Actualmente, los autores más prolíficos desempeñan docencia en otras universidades, salvo un autor que continúa en la UDEP. Los autores tienen la siguiente vinculación laboral: José Luis Requejo Alemán, Universidad Carlos III de Madrid; Lizardo Vargas Bianchi, Universidad de Lima, además, fue primer secretario editorial de la revista; Francisco Arbaiza Rodríguez, Universidad Peruana de Ciencias Aplicadas y Tomás Atarama Rojas, Universidad de Piura. Las autoras que se ubican entre las posiciones de 5 a 9, con 5 citas, también tienen algún tipo de vinculación con la UDEP: egresaron o ejercieron docencia. Con respecto a las citaciones obtenidas por los autores de la revista, una vista a los 19 autores más productivos muestra que aquellos citados al menos una vez son solo 16 en GS, 9 en Scopus y 8 en WoS (Tabla 2). Al realizar una comparación de fuentes de citación para los autores más citados con respecto al total de artículos analizados y las citaciones obtenidas, podemos observar que las citas concentradas en los más productivos acumulan el 17\% para GS (de 987 citas), el $12 \%$ en Scopus (de 169 citas) y $11 \%$ en WoS (de 159 citas).

Por otro lado, la proyección mediante la distribución de autores según su productividad (número de firmas), calculado por la ley del cuadrado inverso de Lotka (Ecuación 1), evidenció que las contribuciones observadas mayores o iguales a 2 artículos por autor son sustancialmente inferiores a los datos proyectados (Figura 1).

\section{Impacto de las publicaciones: citas y h-index}

Para el análisis de citas de la Revista de Comunicación se considera como fuente principal a Google Scholar con una mayor cobertura, incluye artículos, libros, presentaciones, blogs; complementariamente se utilizan Scopus y WoS como índices de citación de referencia mundial. La Tabla 3 muestra que la mayor atribución de citas recibidas proviene de Google Scholar 987 citaciones en total que representan un ratio de 3,39 citas por documento; Scopus recoge 169 citas en total, esto es un ratio de 0,58 citas por documento; WoS presenta 159 citas que es el 0,55 citas por documento. Un acer- 
camiento al comportamiento de los artículos y las citas obtenidas de acuerdo con las tres fuentes de datos utilizadas se realizó mediante el indicador de Hirsch que arroja el mismo dato para Scopus y WoS, y superior en GS (Tabla 3). Adicionalmente, si comparamos las citas recibidas por artículos en las tres fuentes, mediante un análisis del coeficiente de Pearson, se encuentra alta correlación de GS con Scopus y WoS $(\mathrm{p}=0,82)$ y similar aproximación entre Scopus y WoS $(\mathrm{p}=0,93)$.

Figura 1. Distribución de firmas por autor

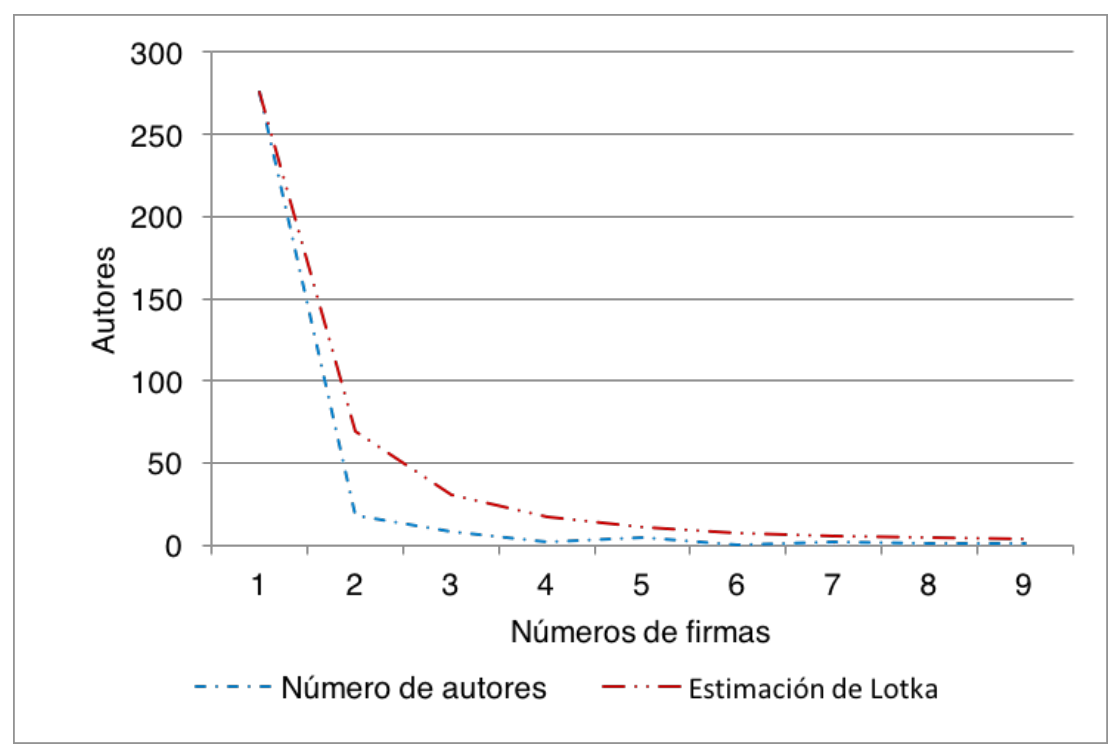

Fuente: elaboración propia

Tabla 3. Indicadores de impacto según fuentes

\begin{tabular}{|l|c|c|c|}
\multicolumn{1}{|c|}{ Fuentes } & Citas & Cit./Doc. & Índice H \\
\hline Scholar Google & 987 & 3,39 & 15 \\
\hline Scopus & 169 & 0,58 & 6 \\
\hline Web of Science & 159 & 0,55 & 6 \\
\hline
\end{tabular}

Fuente: elaboración propia

En la Figura 2 se muestra la evolución de las citaciones recibidas en Google Scholar utilizando ratios de los cocientes de las citas absolutas con respecto a la cantidad de autores y documentos por año. Como se observa, el pico notable corresponde al año 2012 dónde se publicó el artículo más citado y con mayor repercusión de todos los estudiados que obtuvo 117 citas en GS, 33 en Scopus y 23 en WoS, pertenece a Guo et al. (2012) y estudia los efectos de los medios en la percepción de la opinión pública y aplica la Network Agenda Cetina; la revista publica el artículo en inglés y probablemente el idioma haya mejorado su posicionamiento. Lei Guo se desempeña en Boston University, College of Communication; Hong Tien Vu y Maxwell McCombs (emérito) se desempeñan en University of Texas (Austin), School of Journalism (Ver Material Suplementario con lista de autores más citados).

Las colaboraciones entre autores que publicaron en la revista sigue la tendencia predominante de las Ciencias Sociales y las Humanidades: la autoría única (Figura 3). El ratio media total de autoría de la Revista de Comunicación es 1,41 (411firmas de autoría en 291 artículos publicados); el número de autores encontrados de los artículos analizados en la Revista de Comunicación es de 314; donde la mayoría de los artículos tiene firma única, es decir, 206 artículos fueron escritos por un solo autor (71\%); mientras que los artículos con dos autores suman 55 (19\%); los artículos con tres autores 
suman 24 (8\%), y los artículos con cuatro autores suman 6 (2\%). El ratio de colaboración para el año 2002 es cero para 12 artículos en total, es decir, no hay coautorías; posteriormente se observa un ligero y constante incremento hasta 2016, luego de ese año el índice de colaboración presenta incremento sostenido (Figura 3). Desde la vista de autorías sin colaboración, en los dos primeros años (2002-2003) el ratio de colaboración de autorías únicas es el total absoluto (23 artículos), y en 2007 este ratio vuelve a ser igual (15 artículos): sin coautorías. Posteriormente, como se muestra en la Figura 3, las autorías únicas descienden en los siguientes años, en 2014 es de 0,60 (6 de 10 artículos), 2015 de 0,67 ( 8 de 12 artículos), el 2016 asciende a 0,73 (11 de 15 artículos); finalmente, en el año 2019 el ratio de colaboración de autor único es de 0,33 (12 de 36 artículos) o contrariamente el $70 \%$ fue escrito en colaboración (24 artículos).

Figura 2. Crecimiento de citas por autor y documentos en GS

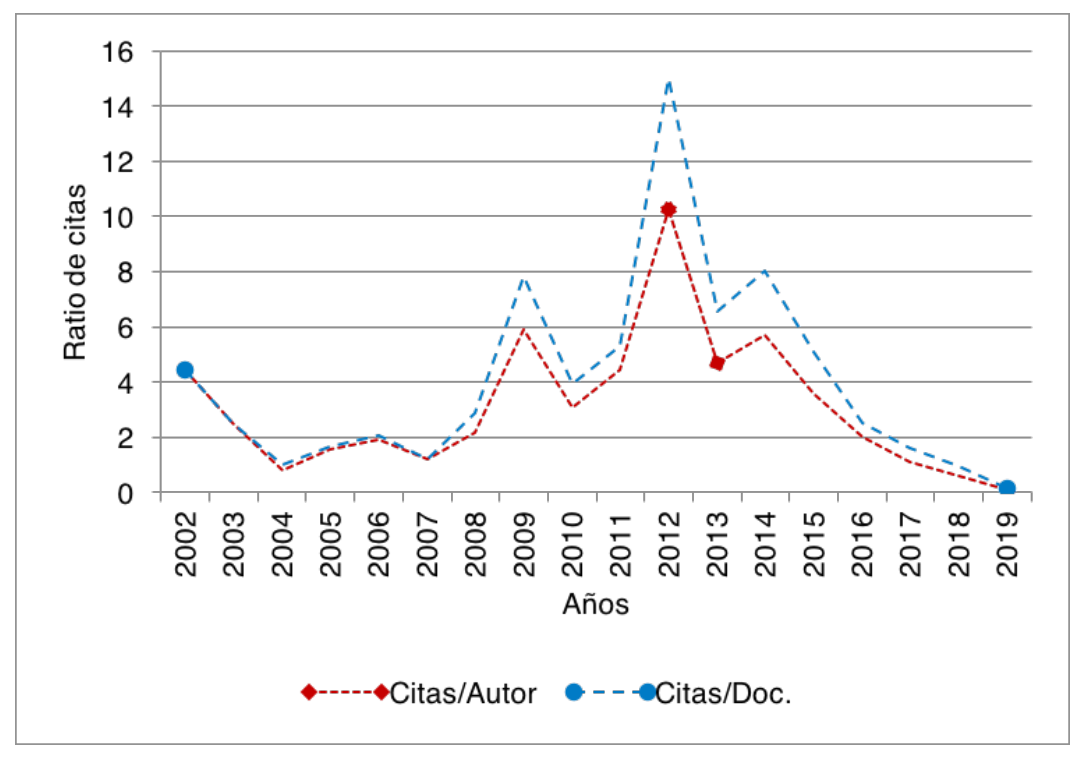

Fuente: elaboración propia

Figura 3. Evolución del índice de colaboración de autores

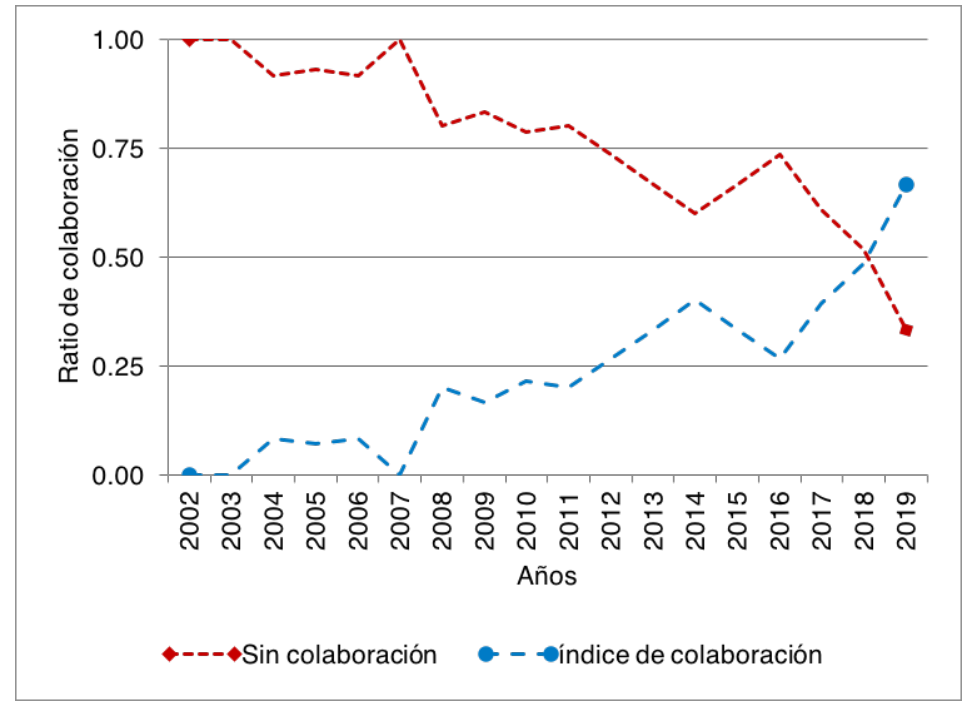

Fuente: elaboración propia 


\section{Tópicos de publicación}

El análisis de ocurrencia de términos (co-word analysis), luego del proceso de normalización y el umbral de frecuencias $(n>1)$ se redujo a 132 términos, que fueron representados mediante los colores en siete conglomerados como se muestran en la Figura 4. Los datos del análisis de co-ocurrencia para identificar los tópicos de publicación representados mediante el gráfico de densidad, muestran los pesos de aparición por conteo binario mediante el tamaño de cada término. En el grupo de términos en rojo conformado por 16 términos, se observa a tres términos frecuentes: "journalism", "communication" y "media", con frecuencia de aparición de 27, 23 y 23 veces, respectivamente. En el conjunto verde, que incluye 10 términos, se aprecia a tres topónimos como predominantes: "España" $(\mathrm{k}=18)$, "América Latina" y "Brasil" $(\mathrm{k}=4)$, junto a los términos "case" ( $\mathrm{k}=16)$ y "news" $(\mathrm{k}=10)$. Los colores azul, amarillo y morado agrupan a nueve términos cada uno, en los que los términos más frecuentes son "television" (k=10), "medium" $(\mathrm{k}=8)$ y "films" ( $\mathrm{k}=8)$, respectivamente. Entre los conjuntos de términos con menor cantidad de términos se encuentran concentrados en el color celeste con 7 items y en naranja con 4 items) (Figura 4).

Figura 4. Visualización por densidad de co-ocurrencia de términos.

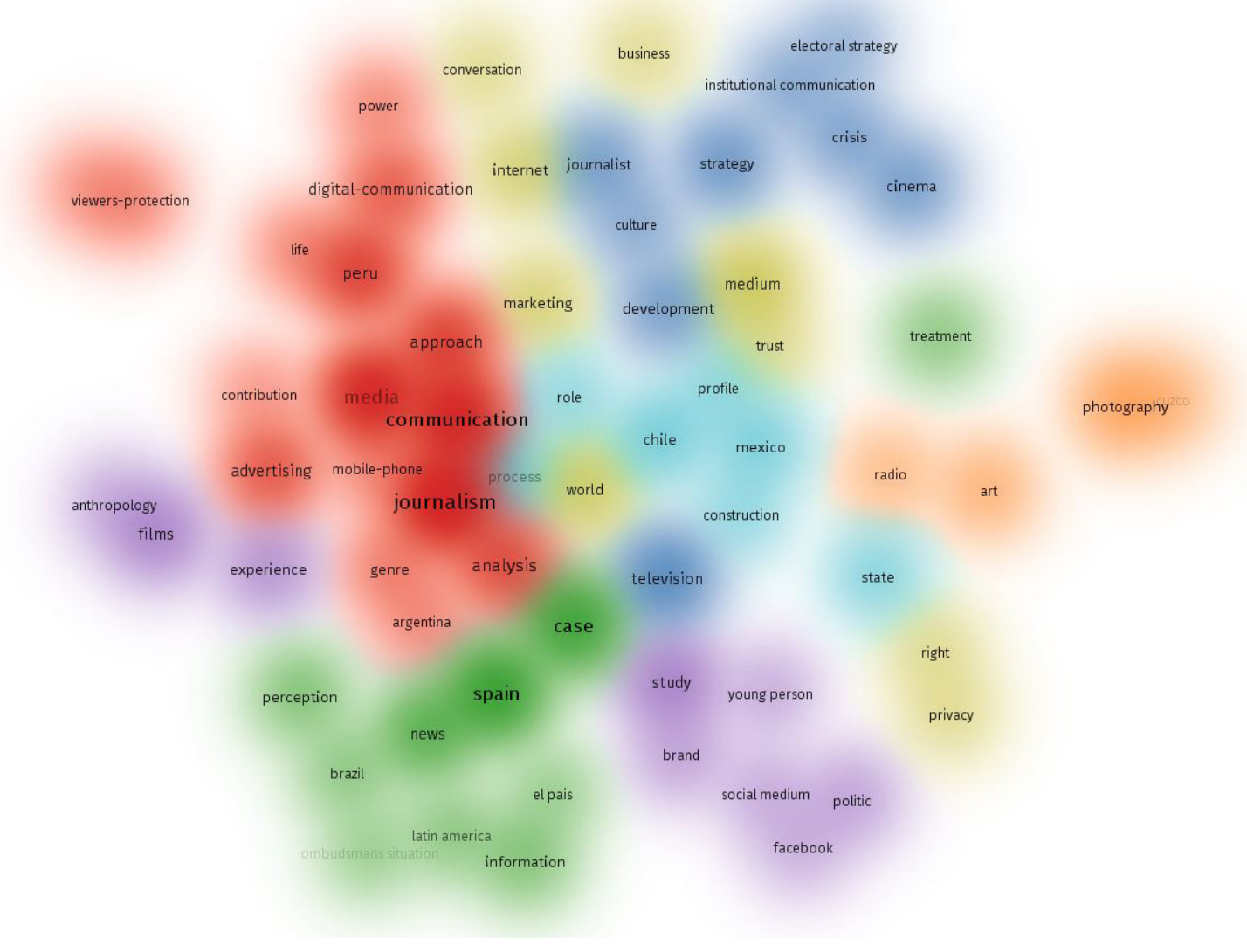

Fuente: elaboración propia

\section{Discusión}

El corpus de artículos de la Revista de Comunicación muestra un crecimiento sostenido en los indicadores de citas y demuestra el posicionamiento de la revista a nivel nacional e internacional, este primer estudio es el derrotero para otras investigaciones sobre la propia revista y su futura evolución en el ámbito académico.

En estudios relacionados con la colaboración de autores similares a los aplicados a la Revista de Comunicación (un solo autor $71 \%$; dos autores $19 \%$; tres autores $8 \%$ y cuatro autores $2 \%$ ), donde se analizan los 
patrones de autoría a partir del caso de una revista muestran hasta un $51 \%$ de documentos firmados por dos autores (Shukla \& Verma, 2019) y un 75\% en autoría única (Jena et al., 2012), cada revista tiene una característica diferente probablemente obedece a diversas formas de asumir el trabajo de investigación.

En la colaboración de autores, el ratio media total de la Revista de Comunicación es menor que en otras investigaciones 1,41 (411firmas de autoría en 291 artículos publicados). Escribà Saler y Cortiñas-Rovira (2013) establecen un ratio de 1,75 por cada trabajo de investigación (2,072 autorías con 1,184 artículos) en revistas de comunicación españolas en un periodo de 2007 a 2011; otro estudio reporta una media de 1,51 (de 1.506 autorías para un millar de artículos) de cinco revistas de comunicación española publicados ente 1990 y 2014 e (Martínez-Nicolás \& Carrasco-Campos, 2018).

Los indicadores de evaluación a nivel de autor y documentos publicados combinados con patrones de autoría y datos de citaciones recibidas resultan elementos pertinentes para la evaluación de revistas como casos particulares de análisis. Por otro lado, la utilización de fuentes de datos comerciales de citas para los artículos que no están indizados permite establecer un panorama de la situación general de la revista en más de un escenario posible; de igual modo, la comparación con resultados provenientes de fuentes libres como Google Scholar amplía la perspectiva del estudio.

Las herramientas de análisis empleados responden a los indicadores y fuentes de datos propuestos; sin embargo, la utilización de estos recursos por sí mismos, no proporcionan resultados fiables y transparentes si no se emplean métodos apropiados para el análisis.

\section{Conclusiones}

La Revista de Comunicación publicada por la Universidad de Piura está posicionada como una de las mejores publicaciones de América Latina y España e integra un pequeño segmento de revistas de comunicación con mejores indicadores en las bases de datos de WoS, Scopus y GS; este prestigio le permite integrar la Escuela de Revisores - Revistas Científicas de Comunicación (https://www.escueladerevisores.com/).

Los autores más productivos tienen o han tenido un vínculo con la Universidad de Piura - UDEP, como docentes o egresados; demuestra su calidad como institución formadora y promotora de investigadores.

Los autores más productivos acumulan el 17\% para GS (de 987 citas), el 12\% en Scopus (de 169 citas) y $11 \%$ en WoS (de 159 citas); las cuatro primeras ubicaciones que muestran a los autores más productivos con 9 a 7 artículos publicados, representan el 10.65\% del total de artículos $(n=291)$

El artículo más citado y con mayor repercusión obtuvo 117 citas en GS, 33 en Scopus y 23 en WoS (Guo et al., 2012), publicado en idioma inglés y probablemente sea un indicador para su impacto, otro factor podría ser el alto número de citas de la autora.

El desempeño de los autores a nivel de colaboración ha evidenciado un notable incremento en años recientes, alcanza hasta el $70 \%$ en 2019 , así como un equilibrado impacto obtenido según las citas recibidas en bases de datos como Scopus y WoS.

El análisis de ocurrencia de términos (co-word analysis) tuvo como resultado 7 conglomerados de términos agrupados por colores y responden a la asociación entre los términos presentes en cada título de los artículos.

\section{Recomendaciones}

Un desafío para la Revista de Comunicación es incrementar su presencia en medios sociales, especialmente en el Twitter o Facebook y para mejorar su visibilidad y posibilitar el análisis de indicadores altmétricos (altmetrics). 
Para el seguimiento de la evolución de las publicaciones académicas es necesario la creación de un observatorio nacional de revistas peruanas, que cumplan los criterios mínimos de calidad y ayuden a los editores nacionales, especialmente universitarios, en la gestión y la toma de decisiones; una de estas primeras iniciativas se puede encontrar en el proyecto denominado Revistas Científicas Peruanas (http://revistasperuanas.info/).

Material suplementario

El conjunto de datos utilizado en el estudio se encuentra publicado y disponible en libre acceso desde https://dx.doi.org/10.6084/m9.figshare.12173226.

\section{Bibliografía}

Aksnes, D. W., Langfeldt, L., \& Wouters, P. (2019). Citations, citation indicators, and research quality: An overview of basic concepts and theories. Sage Open, 9(1), 1-17. https://doi. org $/ 10.1177 / 2158244019829575$

Alperin, J. P., \& Rozemblum, C. (2017). La reinterpretación de visibilidad y calidad en las nuevas políticas de evaluación de revistas científicas. Revista Interamericana de Bibliotecología, 40, 231241. https://doi.org/10.17533/udea.rib.v40n3a04

Borgman, C. L., \& Furner, J. (2002). Scholarly communication and bibliometrics. Annual Review of Information Science and Technology, 36(1), 2-72. https://doi.org/10.1002/aris.1440360102

Castillo-Esparcia, A. (2012). Investigación e investigadores. Las revistas científicas como instrumento de comunicación. Revista de Comunicación Vivat Academia, 14(117E), 1002-1017. https://doi.org/0.15178/va.2011.117E.1002-101

Castillo Esparcia, A., Almansa Martínez, A., \& Álvarez Nobell, A. (2012). Investigación latinoamericana en comunicación. estudio bibliométrico de revistas científicas. ALAIC Revista Latinoamericana de Ciencias de la Comunicación, 9(16). https://www.alaic.org/revista/index.php/alaic/article/view/483

Chávez-Sánchez, H. G. (2020). Calidad de las revistas cientificas electrónicas de comunicación de América Latina y España (Tesis Maestro en Comunicación Social con Mención en Investigación). Universidad Nacional Mayor de San Marcos. Facultad de Letras y Ciencias Humanas. Lima, Perú.

Delgado, E., \& Repiso, R. (2013). El impacto de las revistas de comunicación: comparando Google Scholar Metrics, Web of Science y Scopus. Comunicar, 21(41), 45-52. https://doi. org/10.3916/C41-2013-04

Escribà Saler, E., \& Cortiñas-Rovira, S. (2013). La internacionalización y las coautorías en las principales revistas científicas de Comunicación en España. Comunicar: Revista Cientifica Iberoamericana de Comunicación y Educación, (41), 35-44. https://doi.org/10.3916/C41-2013-03

Fernández-Quijada, D. (2011). Appraising internationality in Spanish communication journals. Journal of Scholarly Publishing, 43(1), 90-109. https://doi.org/10.3138/jsp.43.1.90

Guo, L., Vu, H. T., \& McCombs, M. (2012). An expanded perspective on agenda-setting effects: Exploring the third level of agenda setting. Revista de Comunicación, (11), 51-68. https:// revistadecomunicacion.com/en/articulos/2012/Art051-068.html

He, Q. (1999). Knowledge discovery through co-word analysis. Library Trends, 48(1), 133-159. http:/hdl.handle.net/2142/8267 
Hirsch, J. E. (2005). An index to quantify an individual's scientific research output. Proceedings of the National academy of Sciences, 102(46), 16569-16572. https://doi.org/10.1073/ pnas.0507655102

Jena, K. L., Swain, D. K., \& Sahoo, K. (2012). Annals of Library and Information Studies, 20022010: a bibliometric study. Library Philosophy and Practice, 716. https://digitalcommons.unl.edu/ libphilprac/716/

Laengle, S., Merigó, J. M., Miranda, J., Słowiński, R., Bomze, I., Borgonovo, E., Dyson, R. G., Oliveira, J. F., \& Teunter, R. (2017). Forty years of the European Journal of Operational Research: A bibliometric overview. European Journal of Operational Research, 262(3), 803-816. https://doi. org/https://doi.org/10.1016/j.ejor.2017.04.027

Lochan Jena, K., Swain Dillip, K., \& Sahoo, K. C. (2012). Scholarly communication in Journal of Financial Crime, 2006-2010: a bibliometric study. Journal of Financial Crime, 19(4), 371-383. https://doi.org/10.1108/13590791211266368

Martínez-Nicolás, M., \& Carrasco-Campos, Á. (2018). La transformación de una comunidad científica. Evolución del patrón de autoría de la investigación española sobre comunicación publicada en revistas especializadas (1990-2014). Revista Latina de Comunicación Social, (73), 1368-1383. https://doi.org/10.4185/RLCS-2018-1311

Morales Morante, L. F. (2016). Producción e impacto de las revistas peruanas del ámbito de las Ciencias Sociales en el catálogo Latindex. Investigación Bibliotecológica, 30(69), 179-204. https://doi.org/10.1016/j.ibbai.2016.04.017

Reeves, B., \& Borgman, C. L. (2006). A Bibliometric Evaluation of Core Journals in Communication Research. Human Communication Research, 10(1), 119-136. https://doi. org/10.1111/j.1468-2958.1983.tb00007.x

Sáez, C., \& Barranquero Carretero, A. (2019). Comunicación, desarrollo y cambio social: presencia española en revistas extranjeras de comunicación con mayor factor de impacto en índices SJR y JCR, 1990-2015. IC: Revista Cientifica de Información y Comunicación, 16, 485515. https://hdl.handle.net/11441/91886

Santillán-Aldana, J., Arakaki, M., De la Vega, A., Calderón-Carranza, M., \& Pacheco-Mendoza, J. (2017). Características generales de las revistas científicas peruanas. Revista Española de Documentación Científica, 40(3), 182. https://doi.org/10.3989/redc.2017.3.1419

Shukla, R., \& Verma, M. K. (2019). Research productivity of the journal of knowledge \& communication management (JKCM) from 2011-2017: A bibliometric analysis. International Journal of Information, Library \& society, 8(1), 54-63.

Urbizagastegui Alvarado, R. (2011). La colaboración de los autores en la literatura producida sobre la Ley de Lotka. Ciência da Informação, 40(2). http://revista.ibict.br/ciinf/article/view/1315

Van Eck, N. J., \& Waltman, L. (2020). VOSviewer Manual. Universiteit Leiden; CWTS Meaningful metrics. https://www.vosviewer.com/download/f-33r2.pdf

Vargas Bianchi, L. (2002). Editorial. Revista de Comunicación, 1. https://revistadecomunicacion. com/es/articulos/2002/Editorial.html

Vílchez-Román, C. (2014). Bibliometric factors associated with h-index of Peruvian researchers with publications indexed on Web of Science and Scopus databases. Transinformação, 26, $143-$ 154. https://doi.org/10.1590/0103-37862014000200004 
Vílchez-Román, C., \& Alhuay-Quispe, J. (2016). Use of Text Mining for Understanding Peruvian Students and Faculties' Perceptions on Bibliometrics Training. Proceedings of the 3rd Annual International Symposium on Information Management and Big Data, September 1-3, Cusco-Peru.

Vizoso, Á., Pérez-Seijo, S., \& López, X. (2019). Diez años de investigación en las principales revistas científicas de comunicación. Tendencias y temáticas en las publicaciones de mayor impacto en JCR y SJR. https://doi.org/10.6035/2174-0992.2019.18.12

Yance-Yupari, V. (2018). Uso de Open Journal System en revistas científicas peruanas. Cultura, 32, 353-366. 